\title{
Editorial
}

\section{Una nueva perspectiva teórica de la bibliometría basada en su dimensión histórica y sus referentes temporales}

E 1 desarrollo teórico alcanzado en los estudios métricos de la información documental ha puesto su atención en la definición de las distintas especialidades métricas, así como en las relaciones de (dis)similitudes que existen entre ellas, las cuales han estado determinadas por la singularidad de sus propios objetos y temas de estudio.

Estas especialidades métricas, conocidas como bibliotecometría (Librametry), bibliometría (Bibliometrics), archivometría (Archivometrics) e informetría (Informetrics), así como otras relacionadas con ellas como la cienciometría (Scientometrics), la webmetría (Webometrics) y más recientemente la altmetría (Altmetrics), han tenido como denominador común en su proceso de conceptualización la aplicación de métodos y modelos matemáticos y estadísticos a las actividades bibliotecaria, bibliográfica, archivística, científico informativa, científico investigativa, a las relaciones sociales en la Web y a otras aplicaciones basadas en los medios sociales y una mayor variedad de actividades comunicativas que se realizan en la Red, respectivamente.

Entre estas especialidades métricas destaca la bibliometría por su madurez, tanto en su praxis como en su desarrollo teórico-conceptual. Su tema de estudio ha estado orientado, en lo fundamental, a la identificación de las regularidades cuantitativas presentes en el flujo de información documental y en los procesos de producción y comunicación científica que en estos operan. Sin embargo, esta orientación clásica o descriptiva ha tomado nuevas dimensiones en su propio proceso de maduración, tales como la evaluativa y la histórica, en las que los objetos de estudio tradicionales son suplantados de forma 
complementaria por el enfoque y orientación que se determina en la obtención de sus resultados.

Ante esta disyuntiva un referente a considerar como objeto de estudio fundamental es el tiempo. En analogía con lo que sucede con el análisis demográfico, en el bibliométrico el tiempo determina la orientación o dimensión que puede tomar la obtención de los resultados que de su análisis se derivan. El cambio en el objeto de estudio de la bibliometría al referente temporal nunca ha sido indagado, menos aún la propuesta del desarrollo de una nueva perspectiva teórica de la bibliometría que parta del análisis del referente temporal como eje central en el cual se integren las tres dimensiones hasta ahora identificadas en esta especialidad métrica.

Para sustentar este cambio de enfoque en la perspectiva teórica de la bibliometría es necesario realizar investigaciones orientadas a estos fines. En este sentido, el autor de estas líneas desarrolla un proyecto encaminado a identificar en qué medida la determinación de los referentes temporales de las variables bibliográficas de los documentos contribuye al desarrollo de una nueva perspectiva teórica de la bibliometría, diferente a los enfoques que hasta ahora la han fundamentado como especialidad métrica y que han propiciado el desarrollo de sus dimensiones descriptiva o clásica, evaluativa e histórica. Para lo anterior se identifica y define el carácter tridimensional de la bibliometría a partir de la diversidad de enfoques desarrollados en las investigaciones sobre esta especialidad métrica, al punto de poder determinar un modelo teórico tridimensional propio para la bibliometría.

La bibliometría, como ya se mencionó, constituye la especialidad métrica que más desarrollo conceptual ha tenido y la metría más aplicada; su estudio ha sido el de mayor diversidad temática y cobertura geográfica y temporal de todas las investigaciones realizadas sobre la metría de la información y del conocimiento científico. Propuesta por Otlet en 1934, la bibliometría fue redefinida por Pritchard como "La aplicación de los métodos estadísticos y matemáticos a los libros y otros medios de comunicación". ${ }^{1}$ Esta definición sería retomada y ampliada por este autor en coautoría con Wittig: tation 25 (diciembre 1969) (4): 348. 
incluye todos los estudios que utilizan o discuten análisis estadísticos de datos relacionados con la comunicación impresa [...] estudios de elementos individuales dentro de los trabajos [...] la medición del proceso de transferencia de la Información de su análisis y control. ${ }^{2}$

A las investigaciones bibliométricas realizadas bajo este enfoque teórico o dimensión, en esta primera etapa de desarrollo, se les conoce como descriptivas o clásicas por el uso que hacen de sus modelos matemáticos clásicos, entre ellos los de Lotka, Bradford, Zipf, Price, Brookes, orientados a modelar y determinar niveles de productividad de autores, concentración-dispersión de la información, frecuencia de palabras en los textos, crecimiento exponencial de la ciencia y obsolescencia de las publicaciones científicas, respectivamente.

Otro enfoque teórico que se adiciona a la bibliometría surge en 1976, cuando Narin otorga a esta especialidad su dimensión evaluativa y la define como "el empleo de las técnicas bibliométricas, especialmente el análisis de las publicaciones y las citas, en la evaluación de la actividad científica". 3 Este enfoque, desde nuestro punto de vista, se centra en el estudio de la generación y uso de indicadores bibliométricos orientados a la evaluación de la comunicación y la colaboración entre grupos, comunidades e instituciones científicas. Los resultados obtenidos por la bibliometría evaluativa aportan conocimiento complementario a la interpretación sobre el comportamiento sociológico de las actividades científicas mediante la evaluación de instituciones, comunidades, grupos y recursos humanos dedicados a la actividad científica.

Por último, una tercera dimensión es destacada por Hérubel en 1999 cuando define a la bibliometría histórica como "el estudio de los libros y las revistas enmarcados en tiempo y espacio". 4 Sin embargo, el uso de los referentes temporales y espaciales mencionados en esta definición no constituyen cua-

2 A. Pritchard y G. R. Wittig. Bibliometrics: A Bibliography and Index. Vol.1: 1874-1959. Watford, Herts: ALLM Book, 1981, 3.

3 F. Narin. Evaluative Bibliometrics: The use of publication and citation analysis in the evaluation of scientific activity. Cherry Hill, New Jersey: Computer Horizons, Inc., 1976, 252.

4 J.-P. V. M. Hérubel. "Historical Bibliometrics: Its Purpose and Significance to the History of Disciplines”. Libraries and Culture 34 (Fall 1999) (4): 382. 
lidades privativas de la bibliometría histórica sino que, desde nuestra óptica, resultan imprescindibles en cualquiera de las investigaciones que se realicen en la bibliometría descriptiva, evaluativa o histórica.

La dimensión histórica que se le concede a la bibliometría debiera estar orientada a identificar patrones de comportamiento cuantitativo sobre la historia de la ciencia y los principales hechos que caracterizan el desarrollo científico de una época mediante el uso de las fuentes antiguas y de los indicadores históricos-bibliométricos que identifican sus principales regularidades. Su asociación más significativa, en la orientación de sus resultados, se encuentra identificada con la filosofía y la historia de la ciencia.

Las tres dimensiones que se atribuyen a la bibliometría permiten diseñar un modelo integral orientado a tres enfoques diferentes en la obtención de resultados que también apuntan al uso de fuentes, variables, indicadores y propósitos diferentes y que puede ser representado en un nuevo modelo teórico de la bibliometría. De esta visión surge la intención de proponer un nuevo enfoque en el cual estas tres dimensiones se integren para su análisis mediante el uso del referente temporal ${ }^{5}$ en el cual se consoliden con el propósito de desarrollar una nueva perspectiva teórica en la que el referente temporal constituya el eje principal del análisis bibliométrico, de forma análoga a lo que sucede con el análisis demográfico en los estudios poblacionales.

Las precisiones conceptuales y terminológicas anteriores sobre la referencia temporal de la producción y comunicación científica pueden servir de base para desarrollar una nueva perspectiva teórica de la bibliometría en la que se integren sus tres dimensiones mediante el análisis de los referentes temporales de la producción y comunicación científicas, así como de la argumentación de su dimensión histórica, orientada al aporte de nuevos enfoques que fortalezcan su carácter tridimensional y su análisis integral.

Para la construcción de este nuevo enfoque será necesario determinar, a priori, los aspectos distintivos de las dimensiones descriptiva, evaluativa e histórica de la bibliometría mediante 
el examen de sus (dis)similitudes conceptuales, con el propósito de aportar elementos teóricos que contribuyan a sus redefiniciones; revisar la definición de la bibliometría histórica mediante el análisis del referente temporal en este tipo de estudio para así contribuir con nuevos enfoques a sus bases conceptuales, y establecer el referente temporal como unidad de medida principal en el análisis bibliométrico al aplicar la óptica transversal y longitudinal de los datos, utilizada por los modelos del análisis demográfico.

La construcción de un nuevo enfoque en la perspectiva teórica de la bibliometría debe estar regida por principios básicos. Los documentos y las relaciones de producción y comunicación científica que se manifiestan entre ellos se generan en un momento concreto y perduran en forma y contenido durante un tiempo histórico, por lo que siempre, en forma análoga a lo que ocurre con los individuos que los crean, tienen y cumplen cierta edad cada año de vida. Desde esta perspectiva el tiempo en el análisis bibliométrico constituye la unidad de medida fundamental sobre el cual debe ser identificado el comportamiento de las regularidades de producción y comunicación científicas; el análisis del referente temporal en estas regularidades determina en forma directamente proporcional el análisis de los resultados que se obtienen en las tres dimensiones de la bibliometría y por consiguiente modifican las bases teóricas sobre las cuales, en la actualidad, se sustenta el análisis bibliométrico.

La propuesta de este nuevo enfoque debe iniciar con el examen cuantitativo del efecto que provoca la unidad de medida tiempo en las variables bibliográficas que intervienen en los procesos de producción y comunicación científicas, aspecto muy poco estudiado con antelación en la bibliometría, para al final establecer los eventos que expliquen una nueva perspectiva teórica en esta especialidad métrica. Para ello, se parte del análisis temporal en todos y cada uno de los eventos y regularidades que intervienen en los procesos de producción y comunicación científica (producción, edición, reimpresión, compilación, productividad, concentración, dispersión, envejecimiento, pérdida de utilidad, referenciación, citación, impacto, visibilidad, uso) mediante el diseño transversal, es decir, la recolección de datos en un momento único, y el longitudinal, que consiste en recabar datos en diferentes puntos del tiempo 
para realizar inferencias acerca de la evolución de las causas y sus efectos en los procesos mencionados.

$\mathrm{El}$ análisis y diseño anterior requiere de los tres sistemas de variables que se identifican en el análisis bibliométrico, es decir, las variables de los asientos y registros bibliográficos, las de las referencias y las citas, y las de los autores, grupos, comunidades e instituciones.

El análisis de estos tres sistemas de variables debe realizarse de forma similar a las determinadas por Brookes en el estudio de la obsolescencia: sincrónica, multisincrónica y diacrónica; es decir, retrospectiva en un punto o momento en el tiempo, retrospectiva en múltiples puntos o momentos en el tiempo y proyectiva, hacia el futuro. Para facilitar estos tipos de análisis resulta conveniente la utilización de un modelo heredado del análisis demográfico, que constituye una potente herramienta del análisis cuantitativo en el cual se utiliza el tiempo como unidad de medida determinante, conocido como el Diagrama de Lexis. ${ }^{6}$

Esta interacción entre el análisis bibliométrico y el demográfico comprueba una vez más el carácter transdisciplinario de la metría de la información y del conocimiento científico en general y en particular el de la bibliometría, al tiempo que permite corroborar la fiabilidad del uso de modelos y métodos matemáticos en la determinación de los constructos teóricos que sustentan las investigaciones en este campo de conocimiento.

\section{Salvador Gorbea Portal}

\section{Para citar este texto:}

Gorbea Portal, Salvador. 2016. "Una nueva perspectiva teórica de la bibliometría basada en su dimensión histórica y sus referentes temporales." Investigación Bibliotecológica: Archivonomía, Bibliotecología e Información 70: 11-16. http://dx.doi.org/10.1016/j.ibbai.2016.10.001

\section{$\infty$}

6 J. Pérez Diaz, "El diagrama de Lexis y la referencia temporal de los datos", Apuntes de Demografía (blog). Fecha de consulta: 18 de septiembre de 2015, http://apuntesdedemografia.com/curso-de-demografia/temario/tema-2-gene ralidades/el-diagrama-de-lexis/ 